\title{
POLYHEDRAL GROUPS AND PENCILS OF K3-SURFACES WITH MAXIMAL PICARD NUMBERA*
}

\author{
W. BARTH ${ }^{\dagger}$ AND A. SARTI ${ }^{\ddagger}$
}

\begin{abstract}
A K3-surface is a (smooth) simply-connected surface with trivial canonical bundle. In this note we investigate three particular pencils of K3-surfaces with maximal Picard number. To be precise: The general member in each pencil has Picard number 19. And each pencil contains precisely five surfaces with singularities. Four of them are also singular in the sense that their Picard number is 20 . Our surfaces are minimal resolutions of quotients $X / G$, where $G \subset S O(4, \mathbb{R})$ is a finite group and $X$ a $G$-invariant surface. The singularities of $X / G$ come from fix-points of $G$ on $X$ or from double points of $X$. In any case these singularitites are A-D-E. The rational curves resolving them together with some even, resp. 3-divisible sets of rational curves generate the Neron-Severi group.
\end{abstract}

0. Introduction. The aim of this note is to present three particular pencils of K3-surfaces with Picard-number $\geq 19$. These three pencils are related to the three polyhedral groups $T, O$, resp. $I$, (the rotation groups of the platonic solids tetrahedron, octahedron and icosahedron) as follows: It is classical that the group $S O(4, \mathbb{R})$ contains central extensions

\begin{tabular}{c|ccc} 
& $G_{6}$ & $G_{8}$ & $G_{12}$ \\
\hline of & $T \times T$ & $O \times O$ & $I \times I$
\end{tabular}

by \pm 1 . Each group $G_{n}, n=6,8,12$, has the obvious invariant $q:=x_{0}^{2}+x_{1}^{2}+x_{2}^{2}+x_{3}^{2}$. In [S] it is shown that each group $G_{n}$ admits a second non-trivial invariant $s_{n}$ of degree $n$. (The existence of these invariants seems to have been known before [Ra,C], but not their explicit form as computed in [S].) The pencil

$$
X_{\lambda} \subset \mathbb{P}_{3}(\mathbb{C}): \quad s_{n}+\lambda q^{n / 2}=0
$$

therefore consists of degree- $n$ surfaces admitting the symmetry group $G_{n}$. We consider here the pencil of quotient surfaces

$$
Y_{\lambda}^{\prime}:=X_{\lambda} / G_{n} \subset \mathbb{P}_{3} / G_{n}
$$

It is - for us - quite unexpected that these (singular) surfaces have minimal resolutions $Y_{\lambda}$, which are K3-surfaces with Picard-number $\geq 19$.

In [S] it is shown that the general surface $X_{\lambda}$ is smooth and that for each $n=$ $6,8,12$ there are precisely four singular surfaces $X_{\lambda}, \lambda \in \mathbb{C}$. The singularities of these surfaces are ordinary nodes (double points $A_{1}$ ) forming one orbit under $G_{n}$.

For a smooth surface $X_{\lambda}$ the singularities on the quotient surface $Y_{\lambda}^{\prime}$ originate from fix-points of subgroups of $G_{n}$. Using [S, sect. 7] it is easy to enumerate these fix-points and to determine the corresponding quotient singularities. On the minimal resolution $Y_{\lambda}$ of $Y_{\lambda}^{\prime}$ we find enough rational curves to generate a lattice in $N S\left(Y_{\lambda}\right)$ of rank 19. In sect. 5 we show that the minimal desingularisation $Y_{\lambda}$ is $\mathrm{K} 3$ and that the

${ }^{*}$ Received January 21, 2003; accepted for publication August 12, 2003. Supported by the Schwerpunktprogramm "Global methods in complex geometry" of the Deutsche Forschungsgemeinschaft.

†Universität Erlangen, Mathematisches Institut, Bismarckstrasse 1 1/2, D-91054 Erlangen, Germany (barth@mi.uni-erlangen.de).

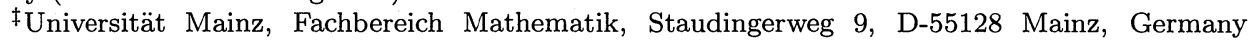
(sarti@Mathematik.Uni-Mainz.DE). 
structure of this surface varies with $\lambda$. This implies that the general surface $Y_{\lambda}$ has Picard number 19. Then in sect. 6.1 we use even sets [N], resp. 3-divisible sets [B, T] of rational curves to determine completely the Picard-lattice of these surfaces $Y_{\lambda}$.

If $X_{\lambda}$ is one of the four nodal surfaces in the pencil, there is an additional rational curve on $Y_{\lambda}$. This surface then has Picard-number 20. (Such K3-surfaces usually are called singular [SI].) We compute the Picard-lattice for the surfaces $Y_{\lambda}$ in all twelve cases (sect. 6.2).

1. Notations and conventions. The base field always is $\mathbb{C}$. We abbreviate complex roots of unity as follows:

$$
\omega=e^{2 \pi i / 3}=\frac{1}{2}(-1+\sqrt{-3}), \quad \epsilon:=e^{2 \pi i / 5}, \quad \gamma:=e^{2 \pi i / 8}=\frac{1}{\sqrt{2}}(1+i) .
$$

By $G \subset S O(3)$ we always denote one of the (ternary) polyhedral groups $T, O$ or $I$, and by $\tilde{G} \subset S U(2)$ the corresponding binary group. By

$$
\sigma: S U(2) \times S U(2) \rightarrow S O(4)
$$

we denote the classical $2: 1$ covering. The group $G_{n} \subset S O(4), n=6,8,12$, is the image $\sigma(\tilde{G} \times \tilde{G})$ for $\tilde{G}=\tilde{T}, \tilde{O}, \tilde{I}$. Usually we are interested more in the group

$$
P G_{n}=G_{n} /\{ \pm 1\} \subset P G L(4) \text {. }
$$

For $n=6,8,12$ it is isomorphic with $T \times T, O \times O, I \times I$ having the order $12^{2}=$ $144,24^{2}=576$, resp. $60^{2}=3600$.

Definition 1.1. a) Let $i d \neq g \in P G_{n}$. A fix-line for $g$ is a line $L \subset \mathbb{P}_{3}$ with $g x=x$ for all $x \in L$. The fix-group $F_{L} \subset P G_{n}$ is the subgroup consisting of all $h \in P G_{n}$ with $h x=x$ for all $x \in L$. The order $o(L)$ of $L$ is the order of this group $F_{L}$.

b) The stabilizer group $H_{L} \subset P G_{n}$ is the subgroup consisting of all $h \in P G_{n}$ with $h L=L$. The length $\ell(L)$ is the length

$$
\left|P G_{n}\right| /\left|H_{L}\right|
$$

of the $G_{n}$-orbit of $L$.

c) We shall encounter fix-lines of orders $2,3,4$ and 5 . We define their types by

$$
\begin{array}{c|cccc}
\text { order } & 2 & 3 & 4 & 5 \\
\hline \text { type } & M & N & R & S
\end{array}
$$

We shall denote by $X_{\lambda}: s_{n}+\lambda q^{n / 2}=0$ the symmetric surface with parameter $\lambda \in \mathbb{C}$. All these surfaces are smooth, but for four parameters $\lambda_{i}$. These four singular parameters in the normalization of $[\mathrm{S}, \mathrm{p} .445, \mathrm{p} .449]$ are

\begin{tabular}{cccc|cccc|cccc}
\multicolumn{4}{c|}{$n=6$} & \multicolumn{5}{c|}{$n=8$} & \multicolumn{4}{c}{$n=12$} \\
\hline$\lambda_{1}$ & $\lambda_{2}$ & $\lambda_{3}$ & $\lambda_{4}$ & $\lambda_{1}$ & $\lambda_{2}$ & $\lambda_{3}$ & $\lambda_{4}$ & $\lambda_{1}$ & $\lambda_{2}$ & $\lambda_{3}$ & $\lambda_{4}$ \\
-1 & $-\frac{2}{3}$ & $-\frac{7}{12}$ & $-\frac{1}{4}$ & -1 & $-\frac{3}{4}$ & $-\frac{9}{16}$ & $-\frac{5}{9}$ & $-\frac{3}{32}$ & $-\frac{22}{243}$ & $-\frac{2}{25}$ & 0
\end{tabular}

Sometimes we call the surface $X_{\lambda}$ of degree $n$ and parameter $\lambda_{i}$ just $X_{n, i}$, or refer to it as the case $n, i$. 
2. Fixpoints. In this section we determine the fix-points for elements $i d \neq g \in$ $P G_{n}$.

Recall that each $\pm 1 \neq p \in \tilde{G}$ has precisely two eigen-spaces in $\mathbb{C}^{2}$ with the product of its eigen-values $=\operatorname{det}(p)=1$.

In coordinates $x_{0}, \ldots, x_{3}$ on $\mathbb{R}^{4}$ the morphism $\sigma: \tilde{G} \times \tilde{G} \rightarrow S O(4, \mathbb{R})$ is defined by $\sigma\left(p_{1}, p_{2}\right):\left(x_{k}\right) \mapsto\left(y_{k}\right)$ with

$$
\left(\begin{array}{cc}
y_{0}+i y_{1} & y_{2}+i y_{3} \\
-y_{2}+i y_{3} & y_{0}-i y_{1}
\end{array}\right)=p_{1} \cdot\left(\begin{array}{cc}
x_{0}+i x_{1} & x_{2}+i x_{3} \\
-x_{2}+i x_{3} & x_{0}-i x_{1}
\end{array}\right) \cdot p_{2}^{-1} .
$$

The quadratic invariant

$$
q=x_{0}^{2}+x_{1}^{2}+x_{2}^{2}+x_{3}^{2}=\operatorname{det}\left(\begin{array}{cc}
x_{0}+i x_{1} & x_{2}+i x_{3} \\
-x_{2}+i x_{3} & x_{0}-i x_{1}
\end{array}\right)
$$

vanishes on tensor-product matrices

$$
\left(\begin{array}{cc}
x_{0}+i x_{1} & x_{2}+i x_{3} \\
-x_{2}+i x_{3} & x_{0}-i x_{1}
\end{array}\right)=\left(\begin{array}{cc}
v_{0} w_{0} & v_{0} w_{1} \\
v_{1} w_{0} & v_{1} w_{1}
\end{array}\right)=v \otimes w
$$

The action of $\tilde{G} \times \tilde{G}$ on the quadric

$$
Q:=\{q=0\}=\mathbb{P}_{1} \times \mathbb{P}_{1}
$$

is induced by the actions of the group $\tilde{G}$ on the tensor factors $v$ and $w \in \mathbb{C}^{2}$

$$
\sigma\left(p_{1}, p_{2}\right): v \otimes w \mapsto\left(p_{1} v\right) \otimes\left(\overline{p_{2}} w\right)
$$

The fix-points for $\pm 1 \neq \sigma\left(p_{1}, p_{2}\right) \in G_{n}$ on $\mathbb{P}_{3}$ come in three kinds:

1) Fix-points on the quadric: $\pm 1 \neq p_{1} \in \tilde{G}$ has two independent eigenvectors $v, v^{\prime}$. The spaces $v \otimes \mathbb{C}^{2}$ and $v^{\prime} \otimes \mathbb{C}^{2}$ determine on the quadric two fix-lines for $\sigma\left(p_{1}, \pm 1\right)$ belonging to the same ruling. In this way $\tilde{G}$-orbits of fix-points for elements $p_{1} \in \tilde{G}$ determine $G_{n}$-orbits of fix-lines in the same ruling of the following lengths:

\begin{tabular}{c|rrrr} 
order of $p$ & 4 & 6 & 8 & 10 \\
\hline$G_{6}$ & 6 & 4,4 & - & - \\
$G_{8}$ & 12 & 8 & 6 & - \\
$G_{12}$ & 30 & 20 & - & 12
\end{tabular}

In the same way fix-points for $p_{2} \in \tilde{G}$ determine fix-lines for $\sigma\left( \pm 1, p_{2}\right) \in G_{n}$ in the other ruling. In [S, p.439] it is shown that the base locus of the pencil $X_{\lambda}$ consists of $2 n$ such fix-lines, $n$ lines in each ruling, say $\Lambda_{k}, \Lambda_{k}^{\prime}, k=1, \ldots, n$. The fix-group $F_{\Lambda_{k}}$ for the general point on each line $\Lambda_{k}, \Lambda_{k}^{\prime}$ then is cyclic of order $s:=|G| / n$ :

$$
\begin{array}{r|rrr}
n & 6 & 8 & 12 \\
\hline s & 2 & 3 & 5
\end{array}
$$

Where a fix-line for $\sigma\left(p_{1}, \pm 1\right)$ meets a fix-line for $\sigma\left( \pm 1, p_{2}\right)$ we obviously have an isolated fix-point $x$ for the group generated by these two symmetries. We denote by $t$ 
the order of the (cyclic) subgroup of $P(\sigma( \pm 1, \tilde{G}))$ fixing $x$. The number of $H_{\Lambda_{k}}$-orbits on each line $\Lambda_{k}$ of such points is

\begin{tabular}{rr|cccc} 
& & \multicolumn{4}{|c}{$t$} \\
$n$ & $s$ & 2 & 3 & 4 & 5 \\
\hline 6 & 2 & 1 & 2 & - & - \\
8 & 3 & 1 & 1 & 1 & - \\
12 & 5 & 1 & 1 & - & 1
\end{tabular}

2) Fix-lines off the quadric: Let $L \subset \mathbb{P}_{3}$ be a fix-line for $\sigma\left(p_{1}, p_{2}\right) \in G_{n}$ with $p_{1}, p_{2} \neq \pm 1$. It meets the quadric in at least one fix-point defined by a tensor product $v \otimes w$ with $v, w$ eigenvectors for $p_{1}, p_{2}$ respectively. The group $<\sigma\left(p_{1}, \pm 1\right)>\subset$ $H_{L}$ centralizes $\sigma\left(p_{1}, p_{2}\right)$. Therefore there is a second fix-point on $L$ for this group. Necessarily it lies on the quadric, being determined by a tensor-product $v^{\prime} \otimes w^{\prime}$ with $v^{\prime}, w^{\prime}$ eigenvectors for $p_{1}, p_{2}$ respectively. Let $\alpha, \alpha^{\prime}$ be the eigen-values for $p_{1}$ on $v, v^{\prime}$ and $\beta, \beta^{\prime}$ those for $p_{2}$ on $w, w^{\prime}$ respectively. Then

$$
\alpha \cdot \alpha^{\prime}=\beta \cdot \beta^{\prime}=1 \text {. }
$$

Since all points on $L$ have the same eigen-value under $\sigma\left(p_{1}, p_{2}\right)$ we find

$$
\alpha \cdot \beta=\alpha^{\prime} \cdot \beta^{\prime}=(\alpha \cdot \beta)^{-1} .
$$

So $\alpha \cdot \beta= \pm 1$ and $g:=\sigma\left(p_{1}, p_{2}\right)$ acts on this line by an eigen-value \pm 1 . In particular $p_{1}$ and $\pm p_{2} \in \tilde{G}$ have the same order.

We reproduce from [S, p. 443] the table of $G_{n}$-orbits of fix-lines off the quadric by specifying a generator $g \in G_{n}$ of $F_{L}$. For this generator we use the notation of [S]. There it is also given the length $\ell(L)$. This length determines the order $\left|H_{L}\right|=$ $\left|P G_{n}\right| / \ell(L)$ of the stabilizer group and the length $\left|H_{L}\right| /\left|F_{L}\right|$ of the general $H_{L^{-}}$orbit on $L$ :

\begin{tabular}{c|ccc|ccccc}
$n$ & \multicolumn{3}{|c|}{6} & \multicolumn{7}{|c}{8} \\
\hline$g$ & $\sigma_{24}$ & $\pi_{3} \pi_{3}^{\prime}$ & $\pi_{3}^{2} \pi_{3}^{\prime}$ & $\pi_{3} \pi_{4} \pi_{3}^{\prime} \pi_{4}^{\prime}$ & $\pi_{3} \pi_{4} \sigma_{4}$ & $\sigma_{2} \pi_{3}^{\prime} \pi_{4}^{\prime}$ & $\pi_{3} \pi_{3}^{\prime}$ & $\pi_{4} \pi_{4}^{\prime}$ \\
$F_{L}$ & $\mathbb{Z}_{2}$ & $\mathbb{Z}_{3}$ & $\mathbb{Z}_{3}$ & $\mathbb{Z}_{2}$ & $\mathbb{Z}_{2}$ & $\mathbb{Z}_{2}$ & $\mathbb{Z}_{3}$ & $\mathbb{Z}_{4}$ \\
type & $M$ & $N$ & $N^{\prime}$ & $M$ & $M^{\prime}$ & $M^{\prime \prime}$ & $N$ & $R$ \\
$\ell(L)$ & 18 & 16 & 16 & 72 & 36 & 36 & 32 & 18 \\
$\left|H_{L}\right| /\left|F_{L}\right|$ & 4 & 3 & 3 & 4 & 8 & 8 & 6 & 8
\end{tabular}

\begin{tabular}{c|ccc}
$n$ & \multicolumn{3}{|c}{12} \\
\hline$g$ & $\sigma_{24}$ & $\pi_{3} \pi_{3}^{\prime}$ & $\pi_{5} \pi_{5}^{\prime}$ \\
$F_{L}$ & $\mathbb{Z}_{2}$ & $\mathbb{Z}_{3}$ & $\mathbb{Z}_{5}$ \\
type & $M$ & $N$ & $S$ \\
$\ell(L)$ & 450 & 200 & 72 \\
$\left|H_{L}\right| /\left|F_{L}\right|$ & 4 & 6 & 10
\end{tabular}

3) Intersections of fix-lines off the quadric: From [S, p.450] one can read off the $G_{n}$-orbits of intersections of these lines outside of the quadric and the value of the parameter $\lambda$ for the surface $X_{\lambda}$ passing through this intersection point. An intersection point is a fix-point for the group generated by the transformations leaving 
fixed the intersecting lines. In the following table we give these (projective) groups $\left(D_{n}\right.$ denoting the dihedral group of order $2 n$ ), the orders of the fix-group of intersecting lines, the generators of these groups, as well as the numbers of lines meeting:

\begin{tabular}{r|r|llll}
$n$ & $\lambda$ & group & orders & generators & numbers \\
\hline 6 & $\lambda_{1}$ & $T$ & 2,3 & $\sigma_{24}, \pi_{3} \pi_{3}^{\prime}$ & 3,4 \\
& $\lambda_{4}$ & $T$ & 2,3 & $\sigma_{24}, \pi_{3}^{2} \pi_{3}^{\prime}$ & 3,4 \\
\hline 8 & $\lambda_{1}$ & $O$ & $2,3,4$ & $\pi_{3} \pi_{4} \pi_{3}^{\prime} \pi_{4}^{\prime}, \pi_{3} \pi_{3}^{\prime}, \pi_{4} \pi_{4}^{\prime}$ & $6,4,3$ \\
& $\lambda_{2}$ & $D_{4}$ & $2,2,4$ & $\pi_{3} \pi_{4} \sigma_{4}, \sigma_{2} \pi_{3}^{\prime} \pi_{4}^{\prime}, \pi_{4} \pi_{4}^{\prime}$ & $2,2,1$ \\
& $\lambda_{3}$ & $\mathbb{Z}_{2} \times \mathbb{Z}_{2}$ & $2,2,2$ & $\pi_{3} \pi_{4} \sigma_{4}, \sigma_{2} \pi_{3}^{\prime} \pi_{4}^{\prime}, \pi_{3} \pi_{4} \pi_{3}^{\prime} \pi_{4}^{\prime}$ & $1,1,1$ \\
& $\lambda_{4}$ & $D_{3}$ & 2,3 & $\pi_{3} \pi_{4} \pi_{3}^{\prime} \pi_{4}^{\prime}, \pi_{3} \pi_{3}^{\prime}$ & 3,1 \\
\hline 12 & $\lambda_{1}$ & $T$ & 2,3 & $\sigma_{24}, \pi_{3} \pi_{3}^{\prime}$ & 3,4 \\
& $\lambda_{2}$ & $D_{3}$ & 2,3 & $\sigma_{24}, \pi_{3} \pi_{3}^{\prime}$ & 3,1 \\
& $\lambda_{3}$ & $D_{5}$ & 2,5 & $\sigma_{24}, \pi_{5} \pi_{5}^{\prime}$ & 5,1 \\
& $\lambda_{4}$ & $I$ & $2,3,5$ & $\sigma_{24}, \pi_{3} \pi_{3}^{\prime}, \pi_{5} \pi_{5}^{\prime}$ & $15,10,6$
\end{tabular}

3. Quotient singularities. Singularities in the quotient surface $Y^{\prime}=Y_{\lambda}^{\prime}$ originate from fix-points of the group action (or from singularities on $X$, but the latter are included in the fix-points, see $[S,(6.4)])$. We distinguish four types of fix-points on $X=X_{\lambda}$ for elements of $G_{n}$ :

1) Points of the base locus $\Lambda$ of the pencil, $n$ lines in each of the two rulings of the invariant quadric $Q$, the (projective) fix-group being $\mathbb{Z}_{s}$ from section 1 ;

2) points on a line $\Lambda_{k}$ or $\Lambda_{k}^{\prime}$ in the base locus, fixed by the group $\mathbb{Z}_{s}=<\sigma(p, 1)>$ from section 1 and by some non-trivial subgroup $\mathbb{Z}_{t} \subset P(\sigma(1, \tilde{G}))$;

3 ) isolated fixed points on the intersection of a fix-line and a smooth surface $X_{\lambda}$;

4) nodes of a surface $X_{\lambda}$.

1) All points of $\Lambda_{i}$ are fixed by the cyclic group $\mathbb{Z}_{s}$ from section 1 . The quotient map here is a cyclic covering of order $s$. The quotient by $\mathbb{Z}_{s}$ is smooth.

2) Since $G_{n}$ acts on $\Lambda_{i}$ as the ternary polyhedral group $G$, there are orbits of points on $\Lambda_{i}$, fixed under some none-trivial subgroup of $G$. We have to disinguish two cases:

Case 1: The $n$ points, where the line $\Lambda_{i}$ meets some line $\Lambda_{k}^{\prime} \subset \Lambda$. Here the stabilizer group is $\mathbb{Z}_{s} \times \mathbb{Z}_{s}$ acting on $X$ by reflections in the two lines $\Lambda_{i}, \Lambda_{k}^{\prime}$. In such points the quotient surface $Y^{\prime}$ is smooth.

Case 2: The fix-points of other non-trivial subgroups of $G$. The lengths of these orbits and their stabilizer subgroups $\mathbb{Z}_{t} \subset G$ are given in section 1 :

\begin{tabular}{c|rrr}
$t$ & 2 & 3 & 4 \\
\hline$G_{6}$ & - & 4,4 & - \\
$G_{8}$ & 12 & - & 6 \\
$G_{12}$ & 30 & 20 & -
\end{tabular}

The total stabilizer is the direct product $\mathbb{Z}_{s} \times \mathbb{Z}_{t}$. Let $v, v^{\prime}$ be eigen-vectors for $\mathbb{Z}_{s}$ and $w, w^{\prime}$ eigen-vectors for $\mathbb{Z}_{t}$. Let $v \otimes w$ determine the fix-point in question. The surface $X$ is smooth there, containing the line $\mathbb{P}\left(v \otimes \mathbb{C}^{2}\right)$, and intersecting the quadric $Q$ transversally. This implies that the tangent space of $X$ is the plane

$$
y_{0} \cdot v \otimes w+y_{1} \cdot v \otimes w^{\prime}+y_{2} \cdot v^{\prime} \otimes w^{\prime}, \quad y_{0}, y_{1}, y_{2} \in \mathbb{C} .
$$

Let $\sigma\left(p_{1}, \pm 1\right) \in \mathbb{Z}_{s}$ and $\sigma\left( \pm 1, p_{2}\right) \in \mathbb{Z}_{t}$ be generators. Let them act by

$$
\sigma\left(p_{1}, 1\right) v=\alpha v, \sigma\left(p_{1}, 1\right) v^{\prime}=\alpha^{-1} v^{\prime}, \sigma\left(1, p_{2}\right) w=\beta w, \sigma\left(1, p_{2}\right) w^{\prime}=\beta^{-1} w^{\prime} .
$$


These transformations act on the coordinates $y_{\nu}$ of the tangent plane as

\begin{tabular}{c|ccccc} 
& $y_{0}$ & $y_{1}$ & $y_{2}$ & $z_{1}:=y_{1} / y_{0}$ & $z_{2}:=y_{2} / y_{0}$ \\
\hline$\sigma\left(p_{1}, 1\right)$ & $\alpha$ & $\alpha$ & $\alpha^{-1}$ & 1 & $\alpha^{-2}$ \\
$\sigma\left(1, p_{2}\right)$ & $\beta$ & $\beta^{-1}$ & $\beta^{-1}$ & $\beta^{-2}$ & $\beta^{-2}$
\end{tabular}

We introduce local coordinates on $X$ in which the group acts as on $z_{1}, z_{2}$, and in fact use again $z_{1}, z_{2}$ to denote these local coordinates on $X$. We locally form the quotient $X /\left(\mathbb{Z}_{s} \times \mathbb{Z}_{t}\right)$ dividing first by the action of $\mathbb{Z}_{s}$

$$
\left(z_{1}, z_{2}\right) \mapsto\left(z_{1}^{s}, z_{2}\right)
$$

Then we trace the action of $\mathbb{Z}_{t}$ on $z_{1}^{s}$ and $z_{2}$. A generator $\sigma\left(1, p_{2}\right)$ of $\mathbb{Z}_{t}$ acts by

\begin{tabular}{ccccc}
$y_{0}$ & $y_{1}$ & $y_{2}$ & $z_{1}$ & $z_{2}$ \\
\hline$\omega$ & $\omega^{2}$ & $\omega^{2}$ & $\omega$ & $\omega$ \\
$i$ & $-i$ & $-i$ & -1 & -1 \\
$\gamma$ & $\gamma^{7}$ & $\gamma^{7}$ & $-i$ & $-i$
\end{tabular}

The resulting singularities on $Y^{\prime}$ are

\begin{tabular}{c|c|cccc|c}
$n$ & $s$ & $z_{1}$ & $z_{2}$ & $z_{1}^{s}$ & $z_{2}$ & quotient singularity \\
\hline 6 & 2 & $\omega$ & $\omega$ & $\omega^{2}$ & $\omega$ & $A_{2}$ \\
\hline 8 & 3 & -1 & -1 & -1 & -1 & $A_{1}$ \\
& & $-i$ & $-i$ & $i$ & $-i$ & $A_{3}$ \\
\hline 12 & 5 & -1 & -1 & -1 & -1 & $A_{1}$ \\
& 5 & $\omega$ & $\omega$ & $\omega^{2}$ & $\omega$ & $A_{2}$
\end{tabular}

3) Let $L \subset \mathbb{P}_{3}$ be a fix-line for $\sigma\left(p_{1}, p_{2}\right) \in G_{n}$, not lying on the quadric. Assume that $\sigma\left(p_{1}, p_{2}\right)$ is chosen as a generator for the group $F_{L}$. By sect. 1 there are eigenvectors $v, v^{\prime}$ for $p_{1}$ with eigen-values $\alpha, \alpha^{-1}$ and $w, w^{\prime}$ for $p_{2}$ with eigen-values $\beta, \beta^{-1}$ satisfying

$$
\alpha \beta= \pm 1, \quad \alpha \beta=\alpha^{-1} \beta^{-1}= \pm 1
$$

such that $L$ is spanned by $v \otimes w$ and $v^{\prime} \otimes w^{\prime}$. The general surface $X$ meets this line in $n$ distinct points. If the line has order $s$, two of these points lie on the base locus $\Lambda$. So the number of points not in the quadric $Q$ cut out on $L$ by $X$ is

\begin{tabular}{c|rr|rrr|rrr}
$n$ & \multicolumn{2}{|c|}{6} & & 8 & & 12 & \\
\hline$o(L)$ & 2 & 3 & 2 & 3 & 4 & 2 & 3 & 5 \\
number & 4 & 6 & 8 & 6 & 8 & 12 & 12 & 10
\end{tabular}

These points fall into orbits under the stabilizer group $H_{L}$. The lengths of these orbits are given in sect. 1 .

To identify the quotient singularity we have to trace the action of $\sigma\left(p_{1}, p_{2}\right)$ on the tangent plane $T_{x}(X)$. For general $X$ this plane will be transversal to $L$. So it must be the plane spanned by $x, v \otimes w^{\prime}, v^{\prime} \otimes w$. By continuity this then is the case also for all smooth $X$. In particular, all smooth $X$ meet $L$ in $n$ distinct points, i.e., the intersections always are transversal. And by continuity again, the numbers and lengths of $H_{L}$-orbits in $X \cap L$ are the same for all smooth $X$. Since $\sigma\left(p_{1}, p_{2}\right)$ acts

$$
\begin{array}{l|ll}
\text { on } & v \otimes w^{\prime} & v^{\prime} \otimes w \\
\hline \text { by } & \alpha \beta^{-1} & \alpha^{-1} \beta,
\end{array}
$$


the eigen-values for $\sigma\left(p_{1}, p_{2}\right)$ on $T_{x}(X)$ are

$$
\frac{\alpha^{-1} \beta}{\alpha \beta}=\alpha^{-2} \quad \text { and } \quad \frac{\alpha \beta^{-1}}{\alpha \beta}=\beta^{-2}=\left( \pm \frac{1}{\alpha}\right)^{2}=\alpha^{2} .
$$

The resulting quotient singularity on $Y^{\prime}$ therefore is of type $A_{r}$, where $r$ is the order of $L$.

We collect the results in the following table. It shows in each case length and number of $H_{L}$-orbits, the number and type(s) of the quotient singularity(ies).

\begin{tabular}{c|rrr|rrrrr|rrr}
$n$ & \multicolumn{6}{|c|}{6} & \multicolumn{6}{|c|}{8} & \multicolumn{3}{|c}{12} \\
\hline$o(L)$ & 2 & 3 & 3 & 2 & 2 & 2 & 3 & 4 & 2 & 3 & 5 \\
type & $M$ & $N^{\prime}$ & $N^{\prime \prime}$ & $M^{\prime}$ & $M^{\prime \prime}$ & $M$ & $N$ & $R$ & $M$ & $N$ & $S$ \\
length & 4 & 3 & 3 & 8 & 8 & 4 & 6 & 8 & 4 & 6 & 10 \\
number & 1 & 2 & 2 & 1 & 1 & 2 & 1 & 1 & 3 & 2 & 1 \\
singularities & $A_{1}$ & $2 A_{2}$ & $2 A_{2}$ & $A_{1}$ & $A_{1}$ & $2 A_{1}$ & $A_{2}$ & $A_{3}$ & $3 A_{1}$ & $2 A_{2}$ & $A_{4}$
\end{tabular}

4) Finally we consider the nodal surfaces $X$. All the intersections of fix-lines considered in sect. 2 are nodes on the surfaces $X$. There are just two invariant surfaces with nodes not given there, because through their nodes passes just one fix-line. They are $G_{6}$-invariants. Their parameters are as follows:

\begin{tabular}{l|ll}
$\lambda$ & group & generator \\
\hline$\lambda_{2}$ & $\mathbb{Z}_{3}$ & $\pi_{3} \pi_{3}^{\prime}$ \\
$\lambda_{3}$ & $\mathbb{Z}_{3}$ & $\pi_{3} \pi_{3}^{\prime 2}$
\end{tabular}

We use this to collect the data for the twelve singular surfaces $X$ in the next table. We include the number $n s$ of nodes on the surface and specify the group $F \subset P S L(4)$ fixing the node. For each type we give the number of lines meeting in the node. So e.g. $3 M$ means that there are three lines of type $M$ meeting at the node.

\begin{tabular}{c|rrrr|rrrr|rrrr}
$n$ & 6 & & & & 8 & & & & 12 & & & \\
\hline$\lambda$ & $\lambda_{1}$ & $\lambda_{2}$ & $\lambda_{3}$ & $\lambda_{4}$ & $\lambda_{1}$ & $\lambda_{2}$ & $\lambda_{3}$ & $\lambda_{4}$ & $\lambda_{1}$ & $\lambda_{2}$ & $\lambda_{3}$ & $\lambda_{4}$ \\
$n s$ & 12 & 48 & 48 & 12 & 24 & 72 & 144 & 96 & 300 & 600 & 360 & 60 \\
$F$ & $T$ & $\mathbb{Z}_{3}$ & $\mathbb{Z}_{3}$ & $T$ & $O$ & $D_{4}$ & $\mathbb{Z}_{2} \times \mathbb{Z}_{2}$ & $D_{3}$ & $T$ & $D_{3}$ & $D_{5}$ & $I$ \\
\hline & $3 M$ & $1 N^{\prime}$ & $1 N^{\prime \prime}$ & $3 M$ & $6 M$ & $2 M^{\prime}$ & $1 M^{\prime}$ & $3 M$ & $3 M$ & $3 M$ & $5 M$ & $15 M$ \\
& $4 N^{\prime}$ & & & $4 N^{\prime \prime}$ & $4 N$ & $2 M^{\prime \prime}$ & $1 M^{\prime \prime}$ & $1 N$ & $4 N$ & $1 N$ & $1 S$ & $10 N$ \\
& & & & & $3 R$ & $1 R$ & $1 M$ & & & & & $6 S$
\end{tabular}

LEMMA 3.1. Let $G \subset S O(3)$ be a finite subgroup of order $\geq 3$.

a) Up to G-equivariant linear coordinate change, there is a unique G-invariant quadratic polynomial defining a non-degenerate cone with top at the origin.

b) If $X$ is a $G$-invariant surface, having a node at the origin, then there is a $G$-equivariant change of local (analytic) coordinates, such that $X$ is given in the new coordinates by $x^{2}+y^{2}+z^{2}=0$.

Proof. a) We distinguish two cases:

i) $G=\mathbb{Z}_{2} \times \mathbb{Z}_{2}$ generated by the symmetries

$$
(x, y, z) \mapsto(x,-y,-z) \text { and }(x, y, z) \mapsto(-x, y,-z) \text {. }
$$


The quadratic $G$-invariants are generated by the squares $x^{2}, y^{2}$ and $z^{2}$. The invariant polynomial then is of the form $a x^{2}+b y^{2}+c z^{2}$ with $a, b, c \neq 0$. The coordinate change

$$
x^{\prime}:=\sqrt{a} x, y^{\prime}:=\sqrt{b} y, z^{\prime}=\sqrt{c} z
$$

is $G$-equivariant and transforms the polynomial into $x^{\prime 2}+y^{\prime 2}+z^{\prime 2}$.

ii) $G$ contains an element $g$ of order $\geq 3$. Let it act by

$$
(x, y, z) \mapsto(c x-s y, s x+c y, z)
$$

with $c=\cos (\alpha), s=\sin (\alpha)$ and $\alpha \neq 0, \pi$. The quadratic invariants of $g$ are generated by $x^{2}+y^{2}$ and $z^{2}$. The invariant polynomial must be of the form $a\left(x^{2}+y^{2}\right)+b z^{2}$ with $a, b \neq 0$. The $G$-equivariant transformation $x^{\prime}:=\sqrt{a} x, y^{\prime}:=\sqrt{a} y, z^{\prime}:=\sqrt{b} z$ transforms it into the same normal form as in i). This proves the assertion if $G=\langle g\rangle$ is cyclic or if $G$ is dihedral.

In the three other cases $G=T, O$ or $I$, it is well-known that $x^{2}+y^{2}+z^{2}$, up to a constant factor, is the unique quadratic $G$-invariant.

b) Let $X$ be given locally at the origin by an equation $f(x, y, z)=0$ with $f$ some power series. Since $X$ is $G$-invariant, so is the tangent cone of $X$ at the origin. By a) we therefore may assume

$$
f=x^{2}+y^{2}+z^{2}+f_{3}(x, y, z)
$$

with a power series $f_{3}$ containing monomials of degrees $\geq 3$ only. It is well-known that there is a local biholomorphic map $\varphi:(x, y, z) \mapsto\left(x^{\prime}, y^{\prime}, z^{\prime}\right)$ mapping $X$ to its tangent cone, i.e., with the property $\varphi^{*}\left(x^{\prime 2}+y^{\prime 2}+z^{\prime 2}\right)=f$. For the derivative $\varphi^{\prime}(0)$ this implies $\varphi^{\prime}(0)^{*}\left(x^{\prime 2}+y^{\prime 2}+z^{\prime 2}\right)=x^{2}+y^{2}+z^{2}$. After replacing $\varphi$ by $\varphi^{\prime}(0)^{-1} \circ \varphi$ we even may assume $\varphi^{\prime}(0)=i d$.

Now consider the local $G$-equivariant holomorphic map

$$
\Phi:(x, y, z) \mapsto \frac{1}{|G|} \sum_{h \in G} h \circ \varphi \circ h^{-1} .
$$

Using the $G$-invariance of $f$ and $x^{\prime 2}+y^{\prime 2}+z^{\prime 2}$ one easily checks $\Phi^{*}\left(x^{\prime 2}+y^{\prime 2}+z^{\prime 2}\right)=f$. It remains to show, that $\Phi$ locally at the origin is biholomorphic. But this follows from

$$
\Phi^{\prime}(0)=\frac{1}{|G|} \sum_{h \in G} h \circ \varphi^{\prime}(0) \circ h^{-1}=i d .
$$

Now consider the automorphism

$$
\mathbb{C}^{2} \rightarrow \mathbb{C}^{2}, \quad v=\left(v_{0}, v_{1}\right) \mapsto v^{\perp}:=\left(v_{1},-v_{0}\right) .
$$

For $q \in S U(2)$ it is easy to check that $(q v)^{\perp}=\bar{q} v^{\perp}$. Map $\mathbb{C}^{2} \rightarrow \mathbb{C}^{3}$ via $v \mapsto v \otimes v^{\perp}$. Consider $\mathbb{C}^{3}$ as the space of traceless complex matrices

$$
\left(\begin{array}{cc}
i x & y+i z \\
-y+i z & -i x
\end{array}\right)
$$

Then

$$
v \otimes v^{\perp}=\left(\begin{array}{cc}
v_{0} v_{1} & -v_{0}^{2} \\
v_{1}^{2} & -v_{0} v_{1}
\end{array}\right)
$$


is a matrix of determinant $x^{2}+y^{2}+z^{2}=0$. One easily checks that the map $v \mapsto v \otimes v^{\perp}$ is $2: 1$ onto the cone of equation $x^{2}+y^{2}+z^{2}=0$, identifying this cone with the quotient $\mathbb{C}^{2} /<-i d>$. And this map is $S U(2)$-equivariant with respect to the $2: 1$ cover $S U(2) \rightarrow S O(3)$. If $\tilde{G} \subset S U(2)$ is some finite group, then the quotient $\mathbb{C}^{2} / \tilde{G}$ via this map is identified with the quotient of the cone by the corresponding ternary group $G \subset S O(3)$.

Together with lemma 3.1 this shows:

Proposition 3.1. Let $X=X_{\lambda}$ be a nodal surface with $G$ the fix-group $G$ of the node. $T^{2}$,en the image of this node on $X / G_{n}$ is a quotient singularity locally isomorp ${ }^{2}$, with $\mathbb{C}^{2} / \tilde{G}$.

4. Rational curves. We denote by $X=X_{\lambda} \rightarrow Y^{\prime}=Y_{\lambda}^{\prime}$ the quotient map for $G_{n}$ acting on $X$ and by $Y=Y_{\lambda} \rightarrow Y^{\prime}$ the minimal resolution of the quotient singularities on $Y$ coming from the orbits of isolated fixed points in sect. 2. The $n$ lines $\Lambda_{i}, \Lambda_{i}^{\prime} \subset Q$ in each ruling map to one smooth rational curve in $Y^{\prime}$. We denote those by $L, L^{\prime}$. Both these curves meet transversally in a smooth point of $Y^{\prime}$. All quotient singularities are rational double points. Resolving them introduces more rational curves in $Y$. For each singularity $A_{k}$ we get a chain of $k$ smooth rational (-2)-curves. Since the group $\mathbb{Z}_{t}$ from sect. 3 acts on $X$ with $\Lambda_{i}$, resp. $\Lambda_{i}^{\prime}$ defining an eigen-space in the tangent space of $X$, the curves $L, L^{\prime}$ meet the $A_{t-1}$-string in an end curve of this string, avoiding the other curves of the string.

All lines $L$ of the types $M, M^{\prime}, M^{\prime \prime}, N, N^{\prime}, N^{\prime \prime}, R, S$ form one orbit under $G_{n}$. We denote by $M_{i}$ etc. the rational curves resolving the $A_{r}$-singularity on the image of $L \cap X$. If $L \cap X$ consists of more than one $H_{L}$-orbit we get in this way more than one $A_{r}$-configuration of rational curves coming from $L \cap X$.

4.1. The general case. First we consider the quotients of the smooth surfaces $X$ : The striking fact is that the number of the additional rational curves is 17 . We give the dual graphs of the collections of 19 rational curves on $Y$, changing the notation $L, L^{\prime}$ to $L_{3}, L_{3}^{\prime}$ for $n=6,12$ and to $L_{4}, L_{4}^{\prime}$ for $n=8$ :
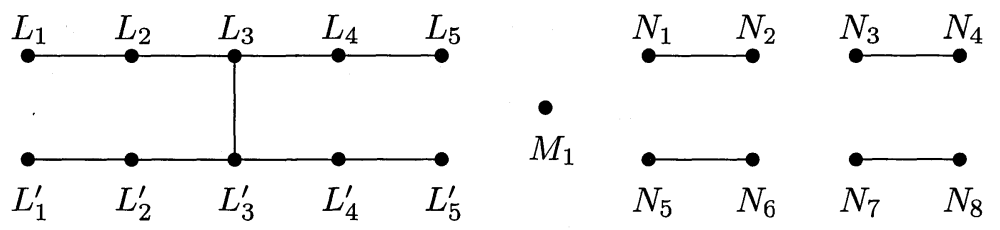

$\mathrm{n}=6: M_{1}$ coming from $M, N_{1}, \ldots, N_{4}$ from $N^{\prime}, N_{5}, \ldots, N_{8}$ from $N^{\prime \prime}$

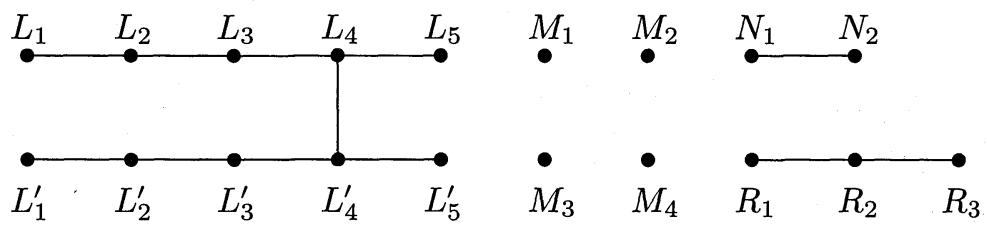

$\mathrm{n}=8: M_{1}$ from $M^{\prime}, M_{2}$ from $M^{\prime \prime}, M_{3}, M_{4}$ from $M, N_{i}$ from $N, R_{i}$ from $R$ 


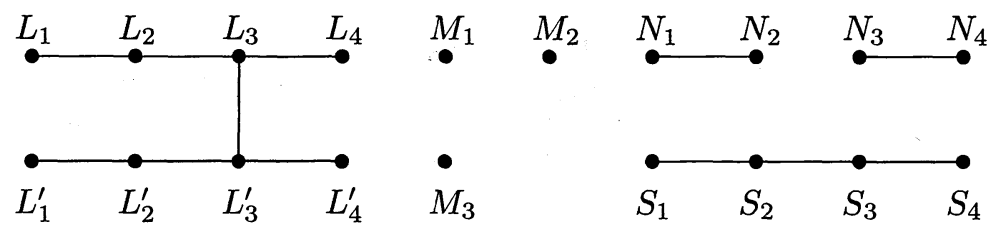

$\mathrm{n}=12: M_{i}$ from $M, N_{i}$ from $N, S_{i}$ from $S$

Proposition 4.1. In each case the 19 rational curves specified generate a sublattice of $N S(Y)$ of rank 19.

Proof. We compute the discriminant $d$ of the lattice. The connected components of the dual graph define sub-lattices, the direct sum of which is the lattice in question. We compute the discriminant block-wise using the sub-lattices

$$
L:=<L_{i}, L_{i}^{\prime}>, \quad M:=<M_{i}>, \quad N:=<N_{i}>, \quad R:=<R_{i}>, \quad S:=<S_{i}>
$$

and find

\begin{tabular}{r|rrrrr|l}
$n$ & $d(L)$ & $d(M)$ & $d(N)$ & $d(R)$ & $d(S)$ & $d$ \\
\hline 6 & -45 & -2 & $3^{4}$ & & & $2 \cdot 3^{6} \cdot 5$ \\
8 & -28 & $2^{4}$ & 3 & -4 & & $2^{8} \cdot 3 \cdot 7$ \\
12 & -11 & $-2^{3}$ & $3^{2}$ & & 5 & $2^{3} \cdot 3^{2} \cdot 5 \cdot 11$
\end{tabular}

4.2. The special cases. Here we consider the desingularized quotients $Y$ for the twelve singular surfaces $X$. The image of the nodes on $X$ will be on $Y$ a quotient singularity for the binary group corresponding to the ternary group $F$ :from sect. 3 . There we also gave the lines passing through this node on $X$. The nodes of $X$ on such a line fall into orbits under the group $H$ fixing the line. If there is just one $H$-orbit of intersection points of the general surface $X$ with this line, it is clear that this orbit converges to the orbit of nodes. We say: The quotient singularity swallows the orbit. If however there are more than one $H$-orbits, we have to analyze the situation more carefully. We use the map onto $\mathbb{P}_{1}$ of this line induced by the parameter $\lambda$. Nodes of $X$ on the given line will be branch points of this map.

Degree 6: On lines of type $M$ there ist just one orbit of four points. On lines of type $N^{\prime}, N^{\prime \prime}$ there are two orbits of length 3 . The parameter $\lambda$ induces on each $N^{\prime}$ - or $N^{\prime \prime}$-line some $6: 1$ cover over $\mathbb{P}_{1}$. Each fibre of six points decomposes into two orbits of three points. The total ramification degree is $-2-6 \cdot(-2)=10$. The intersection with $Q$ consists of two points of ramification order 2. So outside of the quadric $Q$ we will have total ramification order six, hence it will happen twice, that two orbits of three points are swallowed by a quotient singularity. This must happen on the surfaces $X_{6,1}$ and $X_{6,2}$ for $N^{\prime}$, and for $N^{\prime \prime}$ on $X_{6,3}$ and $X_{6,4}$. We give the rational curves from 4.1 disappearing in $Y$, being replaced by rational curves in the minimal resolution of the quotient surface. Here we do not mean that e.g. the curve $N_{1}$ indeed converges to the curve denoted by $N_{1}$ in the dual graph of the resolution. 
We just mean that all the curves denoted by letters in the dual graph disappear:

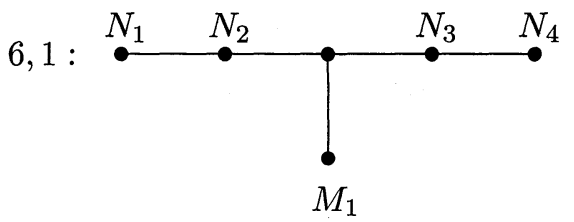

6,2 :

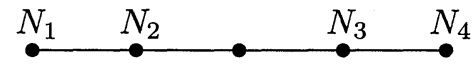

6,3 :

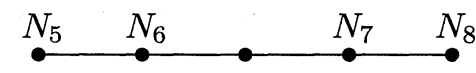

$6,4:$

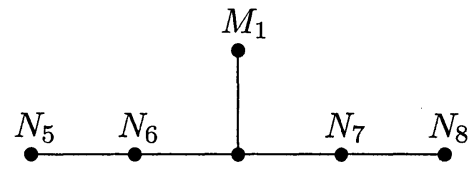

Degree 8: The only lines with two $H$-orbits are those of type $M$. The map to $\mathbb{P}_{1}$ there has degree eight and total ramification order 14 . The intersection with $Q$ counts for two points with ramification order three each. So there will be total ramification of order eight off the quadric. The surface $X_{8,1}$ has $24 \cdot 6 / 72=2$ nodes on such a line, it swallows at least one orbit. The surface $X_{8,3}$ has $144 / 72=2$ nodes too and swallows at least one orbit too. The surface $X_{8,4}$ has $96 \cdot 3 / 72=4$ nodes and swallows at least two orbits. Since the total branching order adds up to at least $2+2+4=8$, the bounds for the numbers of orders in fact are exact numbers. The dual graphs for the resolution of quotient singularities and the curves swallowed are as follows:
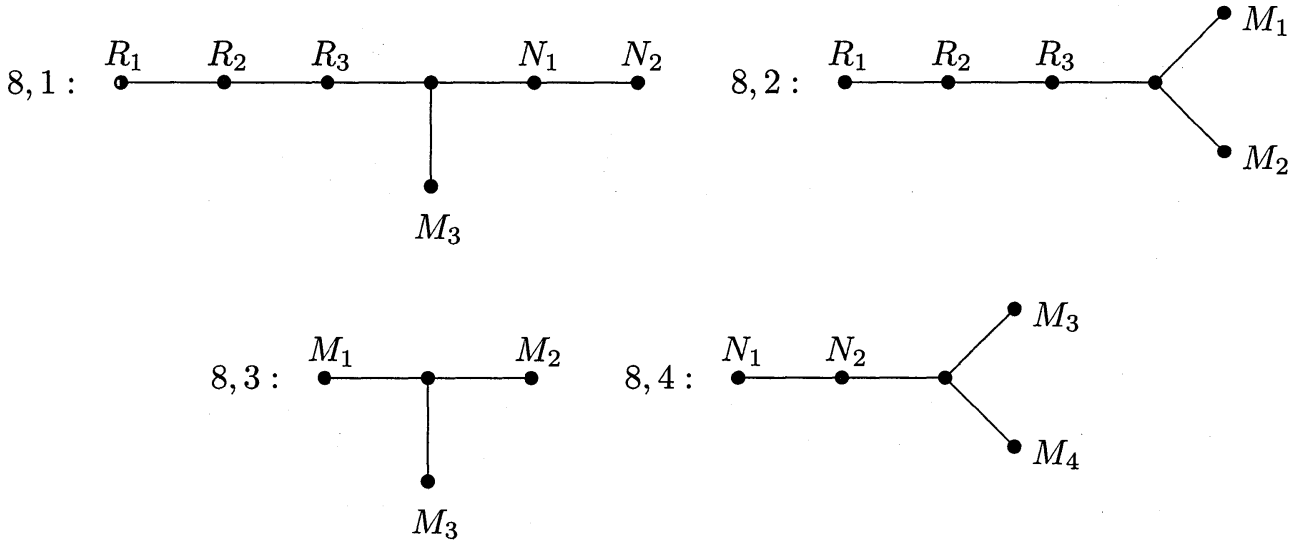

Notice, that it is not necessary here to distinguish between $M_{3}$ and $M_{4}$. In fact it is even impossible, since the two corresponding orbits of intersections of the line $M$ with the surface $X_{\lambda}$ are interchanged by monodromy.

Degree 12: Now a line of type $M$ contains three $H$-orbits of length four. The total branching order for the $\lambda$-map is 22 on such a line. The intersection with $Q$ consists of two six-fold points and decreases the branching order by 10. So the total branching order off the quadric is 12 . On such a line there are

\begin{tabular}{c|cc} 
on the surface & nodes & orbits swallowed \\
\hline$X_{12,1}$ & $300 \cdot 3 / 450=2$ & $\geq 1$ \\
$X_{12,2}$ & $600 \cdot 3 / 450=4$ & $\geq 2$ \\
$X_{12,3}$ & $360 \cdot 5 / 450=4$ & $\geq 2$ \\
$X_{12,4}$ & $60 \cdot 15 / 450=2$ & $\geq 1$
\end{tabular}

Since the total branching order must add up to twelve, the number given is indeed the number of swallowed orbits. 
A line of type $N$ contains two $H$-orbits of length six. Just as in the preceding case one computes the following numbers

\begin{tabular}{c|cc} 
on the surface & nodes & orbits swallowed \\
\hline$X_{12,1}$ & $300 \cdot 4 / 200=6$ & $\geq 2$ \\
$X_{12,2}$ & $600 \cdot 1 / 200=3$ & $\geq 1$ \\
$X_{12,4}$ & $60 \cdot 10 / 200=3$ & $\geq 1$
\end{tabular}

Again the total branching order adds up to twelve. Therefore the estimates give the precise number of orbits swallowed.

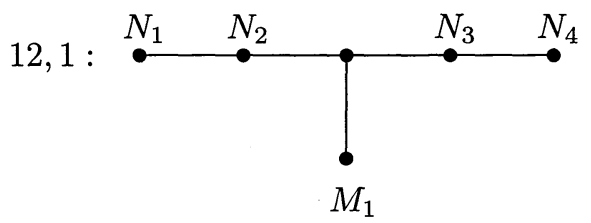

12,2 :

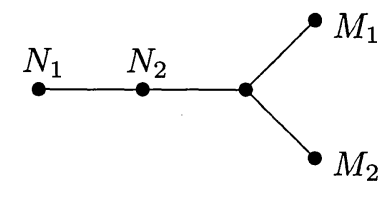

$12,3:$

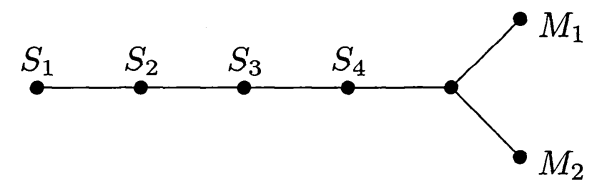

$12,4:$

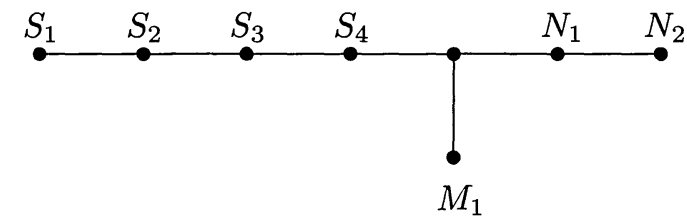

Again, by monodromy it is impossible to distinguish between the curves $M_{1}, M_{2}$ and $M_{3}$, and likewise between the pairs $\left\{N_{1}, N_{2}\right\}$ and $\left\{N_{3}, N_{4}\right\}$.

5. K3-surfaces. In this section we show that the desingularized quotient surfaces $Y_{\lambda}$ are $\mathrm{K} 3$ and that their structure is not constant in $\lambda$. We start with a crude but effective blow-up of $\mathbb{P}_{3}$. Let

$$
\Xi:=\left\{(x, \lambda) \in \mathbb{P}_{3} \times \mathbb{C}: s_{n}(x)+\lambda q^{n / 2}(x)=0\right\} .
$$

In addition we put:

- $\Xi \subset \mathbb{P}_{3} \times \mathbb{P}_{1}$ the closure of $\Xi$. It is a divisor of bidegree (n,1).

- $\tau: \Xi \rightarrow \mathbb{P}_{3}$ the natural projection onto the first factor;

- $f: \Xi \rightarrow \mathbb{C}$ the projection onto the second factor. It is given by the function $\lambda$

- $\tilde{\Lambda}:=\tau^{-1} \Lambda$. This pull-back of the base-locus is the zero-set of $\tau^{*} q$ on $\Xi$;

- $\Xi^{0} \subset \Xi$ the complement of the finitely many points in $\Xi$ lying over the nodes of the four nodal surfaces $X_{\lambda}$.

- $\Upsilon^{\prime}:=\Xi / G_{n}$ the quotient threefold. Notice that the action of $G_{n}$ on $\mathbb{P}_{3}$ lifts naturally to an action on $\Xi$.

- $h: \Upsilon^{\prime} \rightarrow \mathbb{C}$ the map induced by $f$;

- $\Upsilon^{0}$ the image of $\Xi^{0}$. 
LEMma 5.1. a) The threefold $\Xi \subset \mathbb{P}_{3} \times \mathbb{C}$ is smooth.

b) If $M \subset \mathbb{P}_{3}, M \not \subset Q$, is a fix-line for an element $\pm 1 \neq g \in G_{n}$ and $\tilde{M} \subset \Xi$ is its proper transform, then $\tilde{M}$ does not meet $\tilde{\Lambda}$ in $\Xi$.

Proof. a) By $\partial_{\lambda}\left(s_{n}+\lambda q^{n / 2}\right)=q^{n / 2}$ singularities of $\Xi$ can lie only on $\tau^{-1} \Lambda$. But there

$$
\partial_{x_{i}}\left(s_{n}+\lambda q^{n / 2}\right)=\partial_{x_{i}} s_{n}
$$

Since $s_{n}=0$ is smooth along $\Lambda$, this proves that $\Xi$ is smooth.

b) The assertion is obvious, if $M$ does not meet the base locus $\Lambda$. If however $M \cap \Lambda=\left\{x_{1}, x_{2}\right\}$ is nonempty, we use the fact, observed in sect. 3 , that the polynomial $s_{n}+t q^{n / 2} \mid M$ vanishes in $x_{i}$ to the first order for all smooth surfaces $X: s_{n}+t q^{n / 2}=0$. On $\tilde{M}$ however we have $s_{n}=-\lambda q^{n / 2}$ with $n / 2>1$. So $\tilde{M}$ will not meet $\tau^{-1}\left\{x_{1}, x_{2}\right\}$ in $\Xi$.

The $G_{n}$-action on $\Xi$ has the following kinds of fix-points:

1) Fix-points on $\tilde{\Lambda}$ for the group $\mathbb{Z}_{s}$;

2) Fix-points for the group $\mathbb{Z}_{s} \times \mathbb{Z}_{s}$ on the fibre $\tau^{-1}(x)$ over some intersection of lines $\Lambda_{i}, \Lambda_{j}^{\prime}$ in the base locus $\Lambda$;

3) Fix-points for a group $\mathbb{Z}_{s} \times \mathbb{Z}_{t}$ on the fibre $\tau^{-1}(x)$ over a point $x$, where a line in the base locus meets some line $M$ of fix-points not in the base locus. By lemma 5.1 b) $\tau^{-1}(x)$ and $\tilde{M}$ do not intersect in $\Xi$.

4) Fix-curves $\tilde{L}$ away from $\tilde{\Lambda}$ lying over fix-lines $L$ not contained in the baselocus. All these curves are disjoint, when considered in $\Xi^{0}$.

The quotient three-fold $\Upsilon^{\prime}=\Xi / G_{n}$ is smooth in the image points of fix-points of types 1) or 2). It has quotient singularities $A_{t}$ in the image curves of the curves $\tau^{-1}(x)$ of type 3$)$. To be precise: The singularities there locally are products of an $A_{t}$ surface singularity with a copy of the complex unit disc. Additional such cyclic quotient singularities $A_{k}$ occur on the image curves of curves $\tilde{L}$ of type 4 ). Where two such curves meet we have higher singularities. But such points are removed in $\Upsilon^{0}$. So $\Upsilon^{0}$ is singular along finitely many smooth irreducible rational curves. The singularities along each curve are products with some cyclic surface quotient $A_{k}$.

Let $\Upsilon \rightarrow \Upsilon^{0}$ be the minimal desingularisation of $\Upsilon^{0}$ along these singular curves. Locally this is the product of the unit disc with a minimal resolution of the surface singularity $A_{k}$. Since the surfaces $Y_{\lambda}^{\prime}$ intersect the singular curves transversally, the proper transforms $Y_{\lambda} \subset \Upsilon$ are smooth, minimally desingularized. They are the fibres of the map induced by $h$. For $\lambda_{i}, i=1, \ldots, 4$, we denote by $Y_{\lambda_{i}}$ the minimal resolutions of the quotient surfaces $X_{\lambda_{i}} / G_{n}$. We do not (and cannot) consider them as surfaces in $\Upsilon$.

Proposition 5.1. The surfaces $Y_{\lambda}$ are (minimal) K3-surfaces.

Proof. All cyclic quotient singularities on $\Upsilon^{0}$ are gorenstein. So there is a dualizing sheaf $\omega_{\Upsilon 0}$ pulling back to the canonical bundle $K_{\Upsilon}$ on $\Upsilon$. Under the quotient map $\Xi^{0} \rightarrow \Upsilon^{0}$ it pulls back to the canonical bundle $K_{\Xi}$, except for points on the divisor $\tilde{\Lambda}$. There we form the quotient in two steps, as in sect. 3 , first dividing by $\mathbb{Z}_{s}$ and then by $\mathbb{Z}_{t}$. The pull-back via the quotient by $\mathbb{Z}_{t}$ is the canonical bundle of $\Xi / \mathbb{Z}_{s}$. The quotient map for $\mathbb{Z}_{s}$ is branched along $\tilde{\Lambda}$ to the order $s$. So the adjunction formula shows: The dualizing sheaf $\omega_{\Upsilon 0}$ pulls back to

$$
K_{\Xi^{0}} \otimes \mathcal{O}_{\Xi^{0}}((1-s) \tilde{\Lambda})=K_{\Xi^{0}} \otimes \tau^{*}\left(\mathcal{O}_{\mathbb{P}_{3}}(2-2 s)\right)
$$


The divisor $\bar{\Xi} \subset \mathbb{P}_{3} \times \mathbb{P}_{1}$ is a divisor of bi-degree $(n, 1)$. Hence $\bar{\Xi}$ has a dualizing sheaf

$$
\omega_{\bar{\Xi}}=\mathcal{O}_{\mathbb{P}_{3} \times \mathbb{P}_{1}}(n-4,-2)
$$

Now the miracle happens:

$$
n-4=2 s-2
$$

This implies: The pull-back of $\omega_{\Upsilon}$ to $\Xi^{0}$ equals the restriction of $\mathcal{O}_{\mathbb{P}_{3} \times \mathbb{P}_{1}}(0,-2)$, i.e. it is trivial on $\Xi^{0}$.

We distinguish two cases:

a) $\lambda \neq \lambda_{i}, i=1, \ldots, 4$ : The adjunction formula for $Y^{\prime}=Y_{\lambda}^{\prime}=X_{\lambda} / G_{n}$ shows

$$
\omega_{Y^{\prime}}=\omega_{\Upsilon 0} \mid Y^{\prime}
$$

So the pull-back of $\omega_{Y^{\prime}}$ to $X$ is trivial. This implies: $\operatorname{deg}\left(\omega_{Y^{\prime}}\right) \mid C=0$ for all irreducible curves $C \subset Y^{\prime}$ and then $\operatorname{deg}\left(K_{Y} \mid C\right)=0$ for all irreducible curves $C \subset Y$. The surfaces $Y$ have canonical bundles, which are numerically trivial. In particular those surfaces are all minimal. By the classification of algebraic surfaces [BPV p. 168] they are abelian, K3, hyper-elliptic or Enriques. Since we specified in sect. 6.1 rational curves on $Y$ spanning a lattice of rank 19 in $N S(Y)$ the only possibility is $\mathrm{K} 3$.

b) $\lambda=\lambda_{i}, i=1, \ldots, 4$ : The proof of a) shows $\operatorname{deg}\left(K_{Y} \mid C\right)=0$ for all irreducible curves $C \subset Y$ not passing through the exceptional locus of the minimal desingularization $Y \rightarrow Y^{\prime}$. In particular this holds for all curves $C$ which are proper transforms of ample curves $D \subset Y^{\prime}$. Now an arbitrary curve $C \subset Y$ is linearly equivalent to $E+C_{1}-C_{2}$ with $E$ exceptional and $C_{i}$ proper transforms of ample curves $D_{i} \subset Y^{\prime}$. Since all singularities on $Y^{\prime}$ are rational double points of type A,D,E, we have $K_{Y} \cdot E=0$. The method from a) then applies here too.

Proposition 5.2. The structure of the K3-surfaces $Y_{\lambda}$ varies with $\lambda$.

Proof. We restrict to surfaces near some surface $Y$, with $Y^{\prime}$ the quotient of a smooth surface $X$. Here we may assume that the total space $\Upsilon$ is smooth. If all surfaces near $Y$ were isomorphic, locally near $Y$ the fibration would be trivial [FG]. I.e., there would be an isomorphism $\Phi: Y \times D \rightarrow \Upsilon$ respecting the fibre structure. Here $D$ is a copy of the complex unit disc. By the continuity of the induced map

$$
Y=Y \times \lambda \rightarrow Y_{\lambda}
$$

there is an isomorphism $Y \rightarrow Y_{\lambda}$ mapping the 19 rational curves from sect. 4.1 on $Y$ to the corresponding curves on $Y_{\lambda}, \lambda \in D$. The covering $X \rightarrow Y^{\prime}$ is defined by a subgroup in the fundamental group of the complement in $Y$ of these rational curves. This implies that the isomorphism $Y \rightarrow Y_{\lambda}$ induces an isomorphism of the coverings $X \rightarrow X_{\lambda}$ equivariant with respect to the $G_{n}$-action.

Now this isomorphism must map the canonical bundle $\mathcal{O}_{X}(n-4)$ to the canonical bundle $\mathcal{O}_{X_{\lambda}}(n-4)$. Since the surfaces $X_{\lambda}$ are simply-connected, the isomorphism maps $\mathcal{O}_{X}(1)$ to $\mathcal{O}_{X_{\lambda}}(1)$, i.e., it is given by a projectivity. This is in conflict with the following.

Lemma 5.2. For general $\lambda \neq \mu$ there is no projectivity $\varphi: \mathbb{P}_{3} \rightarrow \mathbb{P}_{3}$ inducing some $G_{n}$-equivariant isomorphism $X_{\lambda} \rightarrow X_{\mu}$. 
Proof. Assume that such an isomorphism $\varphi$ exists. Equivariance means for each $g \in G_{n}$ and $x \in X_{\lambda}$ that $\varphi g(x)=g \varphi(x)$ or $\varphi^{-1} g^{-1} \varphi g(x)=x$. Since $X_{\lambda}$ spans $\mathbb{P}_{3}$ this implies the same property for all $x \in \mathbb{P}_{3}$, i.e., the map $\varphi$ is $G_{n}$-equivariant on all of $\mathbb{P}_{3}$. In particular, if $L \subset \mathbb{P}_{3}$ is a fixline for $g \in G_{n}$, then so is $\varphi(L)$. Then we may as well assume $\varphi(L)=L$. We obtain a contradiction by showing that the point sets $X_{\lambda} \cap L$ and $X_{\mu} \cap L$ in general are not projectively equivalent.

The cases $n=6$ and 12: We use the fix-line $L:=\left\{x_{0}=x_{1}=0\right\}$ of type $M$, fixed under $\sigma_{1,3}=\sigma\left(q_{1}, q_{1}\right)$ (notation of [S, p. 432]). The group $H_{L}$ has order 8 , containing in addition the symmetries $\sigma\left(q_{1}, 1\right)$ and $\sigma\left(q_{1} q_{2}, q_{1} q_{2}\right)$ sending a point $x=\left(0: 0: x_{2}: x_{3}\right) \in L$ to

$$
\sigma\left(q_{1}, 1\right)(x)=\left(0: 0: x_{2}:-x_{3}\right), \quad \sigma\left(q_{1} q_{2}, q_{1} q_{2}\right)(x)=\left(0: 0:-x_{3}: x_{2}\right) .
$$

Omitting the first two coordinates and putting $x_{2}=1, x_{3}=u$, we find that a general $H_{L}$-orbit on $L$ consists of points

$$
(1: u),(1: 1 / u),(1:-u),(1:-1 / u)
$$

The cross-ratio of these four points

$$
C R=\frac{2 u}{u+1 / u}: \frac{1 / u+u}{2 / u}=\frac{4 u^{2}}{\left(1+u^{2}\right)^{2}}
$$

varies with $u$. The intersection of $X_{6, \lambda}$ with $L$ consists of one such orbit, the intersection of $X_{12, \lambda}$ of three orbits. This implies the assertion for $n=6$ and 12 .

The case $n=8$ : Here we use the fix-line $L:=\left\{x_{1}=x_{3}, x_{2}=0\right\}$ of type $M$ for $\pi_{3} \pi_{4} \pi_{3}^{\prime} \pi_{4}^{\prime}$. Again $H_{L}$ has order 8 containing in addition $\pi_{3} \pi_{4}$ and $\sigma\left(q_{1} q_{2}, q_{1} q_{2}\right)$. They send a point $x=(u: 1: 0: 1) \in L$ to

$$
\pi_{3} \pi_{4}(x)=(-2: u: 0: u), \quad \sigma\left(q_{1} q_{2}, q_{1} q_{2}\right)(x)=(u:-1: 0:-1) .
$$

Omitting the coordinates $x_{3}$ and $x_{4}$ we find that a general $H_{L}$-orbit consists of

$$
(u: 1),(-u: 1),(2 / u: 1),(-2 / u: 1)
$$

Their cross-ratio

$$
C R=\frac{u-2 / u}{u+2 / u}: \frac{-u-2 / u}{-u+2 / u}=\frac{\left(u^{2}-2\right)^{2}}{\left(u^{2}+2\right)^{2}}
$$

varies with $u$. The intersection of $X_{8, \lambda}$ consists of two such orbits.

Corollary 5.1. The general K3-surface $Y_{\lambda}$ has Picard-number 19.

6. Picard-Lattices. Here we compute the Picard lattices of our quotient K3surfaces $Y$.

6.1. The general case. Denote by $V \subset H^{2}(Y, \mathbb{Z})$ the rank-19 lattice spanned (over $\mathbb{Z}$ ) by the rational curves from sect. 4.1. For $n=6$ and 8 this lattice $V$ is not the total Picard lattice:

Proposition 6.1. a) ( $n=6$ ) After perhaps interchanging curves $N_{2 i-1}$ and $N_{2 i}$ the two divisor-classes

$$
\begin{aligned}
L & :=L_{1}-L_{2}+L_{4}-L_{5}+N_{1}-N_{2}+N_{3}-N_{4}+N_{5}-N_{6}+N_{7}-N_{8}, \\
L^{\prime} & :=L_{1}^{\prime}-L_{2}^{\prime}+L_{4}^{\prime}-L_{5}^{\prime}+N_{1}-N_{2}+N_{3}-N_{4}-N_{5}+N_{6}-N_{7}+N_{8}
\end{aligned}
$$


are divisible by 3 in $N S(Y)$. Together with $V$ the classes $L / 3$ and $L^{\prime} / 3$ span a rank-19 lattice with discriminant $2 \cdot 3^{2} \cdot 5$.

b) $(n=8)$ After perhaps interchanging $M_{1}$ and $M_{2}$ the two classes

$$
\begin{aligned}
L & :=L_{1}+L_{3}+L_{5}+M_{1}+M_{3}+M_{4}+R_{1}+R_{3}, \\
L^{\prime} & :=L_{1}^{\prime}+L_{3}^{\prime}+L_{5}^{\prime}+M_{2}+M_{3}+M_{4}+R_{1}+R_{3}
\end{aligned}
$$

are divisible by 2 in $N S(Y)$. Together with $V$ the classes $L / 2$ and $L^{\prime} / 2$ span a rank-19 lattice with discriminant $2^{4} \cdot 3 \cdot 7$.

Proof. a) Consider reduction modulo 3

$$
\varphi_{3}: \mathbb{Z}^{22}=H^{2}(Y, \mathbb{Z}) \rightarrow H^{2}\left(Y, \mathbb{F}_{3}\right)=\mathbb{F}_{3}^{22}
$$

Because of

$$
M_{1}^{2}=-2, \quad M_{1} \cdot L_{i}^{\prime}=0, \quad \operatorname{det}\left(L_{i}^{\prime}, L_{j}^{\prime}\right)_{i, j=1, . ., 4}=5,
$$

the images of $M_{1}, L_{1}^{\prime}, L_{2}^{\prime}, L_{3}^{\prime}, L_{4}^{\prime}$ span a subspace of $H^{2}\left(Y, \mathbb{F}_{3}\right)$ on which the intersection form has rank 5. The orthogonal complement $C$ of this lattice in $H^{2}\left(Y, \mathbb{F}_{3}\right)$ has dimension 17 and the form is non-degenerate there. This $C$ contains the classes mod 3 of the twelve curves

$$
L_{1}, L_{2}, L_{4}, L_{5}, N_{1}, \ldots, N_{8}
$$

Assume that

$$
D_{1}:=\varphi_{3}<L_{1}, L_{2}, L_{4}, L_{5}, N_{1}, \ldots, N_{8}>
$$

has $\mathbb{F}_{3}$-dimension 12 . Then

$$
D_{2}:=\varphi_{3}<L_{1}-L_{2}, L_{4}-L_{5}, N_{1}-N_{2}, N_{3}-N_{4}, N_{5}-N_{6}, N_{7}-N_{8}>
$$

has dimension six. Since $D_{1} \perp D_{2}$, this is a contradiction. We have shown: A nontrivial linear combination of the twelve classes $L_{1}, L_{2}, L_{4}, L_{5}, N_{1}, \ldots, N_{8}$ lies in the kernel of $\varphi_{3}$. By $[\mathrm{T}]$ such a 3 -divisible class contains at least 12 curves. Hence we may assume the class is

$$
L:=\lambda_{1}\left(L_{1}-L_{2}\right)+\lambda_{4}\left(L_{4}-L_{5}\right)+\sum \nu_{i}\left(N_{2 i-1}-N_{2 i}\right)
$$

with $\lambda_{j}, \nu_{i}= \pm 1$ modulo 3 . W.l.o.g. we put $\lambda_{1}=1$. Intersecting with $L_{3}$ we find $\lambda_{4}=1$ too. And after perhaps interchanging curves $N_{2 i-1}$ with $N_{2 i}$ we may assume $\nu_{1}=\ldots=\nu_{4}=1$.

Exactly in the same way we find a class

$$
L^{\prime}:=L_{1}^{\prime}-L_{2}^{\prime}+L_{4}^{\prime}-L_{5}^{\prime}+\sum \nu_{i}^{\prime}\left(N_{2 i-1}-N_{2 i}\right), \quad \nu_{i}^{\prime}= \pm 1 \bmod 3,
$$

which is 3-divisible in $N S(Y)$. Then $L+L^{\prime}$ is 3-divisible too, and by [T] contains precisely 12 curves. This implies that precisely two coefficients $\nu_{i}^{\prime}$ cancel against the corresponding coefficients of $L$. If these are the coefficients $\nu_{3}^{\prime}$ and $\nu_{4}^{\prime}$, we are done. If this should not be the case, after perhaps interchanging $\left\{N_{1}, N_{2}\right\}$ with $\left\{N_{3}, N_{4}\right\}$, $\left\{N_{5}, N_{6}\right\}$ with $\left\{N_{7}, N_{8}\right\}$ we may assume $\nu_{1}^{\prime}=\nu_{3}^{\prime}=1$ and $\nu_{2}^{\prime}=\nu_{4}^{\prime}=-1$. Denote by $T_{2}: H^{2}(X, \mathbb{Z}) \rightarrow H^{2}(X, \mathbb{Z})$, resp. $H^{2}(Y, \mathbb{Z}) \rightarrow H^{2}(Y, \mathbb{Z})$ the monodromy about $X_{6,2}$ 
(circling the parameter $\lambda_{2}$ in the parameter space) and by $T_{3}$ the monodromy about $X_{6,3}$. So $T_{2}$ interchanges $\left\{N_{1}, N_{2}\right\}$ with $\left\{N_{3}, N_{4}\right\}$, leaving fixed $\left\{N_{5}, N_{6}\right\},\left\{N_{7}, N_{8}\right\}$ with $T_{3}$ doing just the opposite. $N S(Y)$ contains the classes (coefficients modulo 3 )

\begin{tabular}{c|cccccc} 
& $\frac{L_{1}-L_{2}+L_{4}-L_{5}}{3}$ & $\frac{L_{1}^{\prime}-L_{2}^{\prime}+L_{4}^{\prime}-L_{5}^{\prime}}{3}$ & $\frac{N_{1}-N_{2}}{3}$ & $\frac{N_{3}-N_{4}}{3}$ & $\frac{N_{5}-N_{6}}{3}$ & $\frac{N_{7}-N_{8}}{3}$ \\
\hline$L$ & 1 & 0 & 1 & 1 & 1 & 1 \\
$L^{\prime}$ & 0 & 1 & 1 & -1 & 1 & -1 \\
$L+L^{\prime}$ & 1 & 1 & -1 & 0 & -1 & 0 \\
$T_{2}\left(L+L^{\prime}\right)$ & 1 & 1 & 0 & \pm 1 & -1 & 0 \\
$T_{3}\left(L+L^{\prime}\right)$ & 1 & 1 & -1 & 0 & 0 & \pm 1
\end{tabular}

These classes would span in $N S(Y) / V$ a subgroup of order $3^{4}$, in conflict with $d(V)=$ $2 \cdot 3^{6} \cdot 5$, contradiction.

b) Here we consider reduction modulo 2

$$
\varphi_{2}: \mathbb{Z}^{22}=H^{2}(Y, \mathbb{Z}) \rightarrow H^{2}\left(Y, \mathbb{F}_{2}\right)=\mathbb{F}_{2}^{22}
$$

The subspace

$$
C:=\varphi_{2}<L_{1}, L_{3}, L_{5}, M_{1}, M_{2}, M_{3}, M_{4}, R_{1}, R_{3}>\subset H^{2}\left(Y, \mathbb{F}_{2}\right)
$$

is totally isotropic. It is orthogonal to $D:=\varphi_{2}<L_{1}^{\prime}, L_{2}^{\prime}, L_{3}^{\prime}, L_{4}^{\prime}, N_{1}, N_{2}>$. Because of

$$
\operatorname{det}\left(L_{i}^{\prime} \cdot L_{j}^{\prime}\right)_{i, j=1, \ldots, 4}=5, \quad \operatorname{det}\left(N_{i} \cdot N_{j}\right)_{i, j=1,2}=3
$$

the intersection form on $D$ is non-degenerate, and $D^{\perp}$ is non-degenerate of rank 16 . This implies $\operatorname{dim} C \leq 8$. So there is a class

$$
L:=\sum \lambda_{i} L_{i}+\mu_{i} M_{i}+\rho_{i} R_{i}
$$

in the kernel of $\varphi_{2}$. By $[\mathrm{N}]$ it has precisely eight coefficients $=1$. Intersecting

$$
\begin{array}{l|l}
\text { with } & \text { we find } \\
\hline L_{2}, L_{4} & \lambda_{1}=\lambda_{3}=\lambda_{5}=: \lambda \\
R_{2} & \rho_{1}=\rho_{3}=: \rho
\end{array}
$$

This implies that precisely one coefficient $\mu_{i}$ will vanish and $\lambda=\rho=1$. In the same way one finds a class

$$
L^{\prime}:=L_{1}^{\prime}+L_{3}^{\prime}+L_{5}^{\prime}+\sum \mu_{i}^{\prime} M_{i}+R_{1}+R_{3}
$$

in the kernel of $\varphi_{2}$ with precisely one $\mu_{1}^{\prime}$ vanishing. The class

$$
L+L^{\prime}=L_{1}+L_{3}+L_{5}+L_{1}^{\prime}+L_{3}^{\prime}+L_{5}^{\prime}+\sum\left(\mu_{i}+\mu_{i}^{\prime}\right) M_{i}
$$

also is divisible by 2 and has precisely eight non-zero coefficients. It follows that precisely two of the non-zero coefficients from $\mu_{i}$ and $\mu_{i}^{\prime}$ coincide. If $\mu_{3}=\mu_{4}=\mu_{3}^{\prime}=$ $\mu_{4}^{\prime}=1$ we are done (perhaps after interchanging $M_{1}$ and $M_{2}$ ). If this is not the case, assume e.g. $\mu_{1}=\mu_{2}=\mu_{4}=1, \mu_{3}=0$. Denote by $T$ the monodromy about the surface $X_{8,4}$ (circling the parameter $\lambda_{4}$ in the parameter space). It interchanges $M_{3}$ and $M_{4}$. So the two classes

$$
\frac{L}{2}=\frac{1}{2}\left(L_{1}+L_{3}+L_{5}+M_{1}+M_{2}+M_{4}+R_{1}+R_{3}\right)
$$




$$
\frac{T(L)}{2}=\frac{1}{2}\left(L_{1}+L_{3}+L_{5}+M_{1}+M_{2}+M_{3}+R_{1}+R_{3}\right)
$$

would belong to $N S(Y)$. However this contradicts

$$
\frac{L}{2} \cdot \frac{T(L)}{2}=-\frac{7}{2} \notin \mathbb{Z}
$$

TheOREm 6.1. If the Neron-Severi group of $Y$ has rank 19, it is generated by $V$ and

\begin{tabular}{r|l}
$n$ & \\
\hline 6 & $L / 3, L^{\prime} / 3$ \\
8 & $L / 2, L^{\prime} / 2$, \\
12 & no other classes.
\end{tabular}

Proof. Denote by $W \subset N S(Y)$ the lattice spanned by the 19 rational curves from sect. 4.1 and by $L / 3, L^{\prime} / 3$ from prop. 6.1 a) (if $n=6$ ) resp. $L / 2, L^{\prime} / 2$ from prop. $6.1 \mathrm{~b}$ ) (if $n=8$ ). If $N S(Y) \neq W$ there would be an integral lattice $W^{\prime}$ with $W \subset W^{\prime} \subset N S(Y)$ and $p:=\left[W^{\prime}: W\right]$ a prime such that $p^{2}$ divides $d(W)$. The only possibilities are $p=2$ or $=3$. The following table gives in each case generators for the $p$-subgroup $\left(W^{\vee} / W\right)^{p}$ of $W^{\vee} / W$ :

\begin{tabular}{rr|l}
$n$ & $p$ & generators for $\left(W^{\vee} / W\right)^{p}$ \\
\hline 6 & 3 & $\left(N_{1}-N_{2}-N_{3}+N_{4}\right) / 3,\left(N_{5}-N_{6}-N_{7}+N_{8}\right) / 3$ \\
\hline 8 & 2 & $\left(M_{1}+M_{2}+M_{3}\right) / 2,\left(M_{1}+M_{2}+M_{4}\right) / 2$, \\
& & $\left(M_{1}+M_{2}\right) / 2+\left(R_{1}+2 R_{2}+3 R_{3}\right) / 4$ \\
\hline 12 & 2 & $M_{1} / 2, M_{2} / 2, M_{3} / 2$ \\
12 & 3 & $\left(N_{1}-N_{2}\right) / 3,\left(N_{3}-N_{4}\right) / 3$
\end{tabular}

By [N] a divisor consisting of $m$ disjoint rational curves on $Y$ can be divisible by 2 only if $m=8$ or $=16$. For $n=12, p=2$ there are only three such curves, while for $n=8, p=2$ there are only the six curves $M_{1}, M_{2}, M_{3}, M_{4}, R_{1}, R_{3}$. These cases are excluded. By [T] a divisor consisting of $m$ disjoint pairs of rational curves, each pair meeting in one point, is divisible by 3 only if $m=6$ or $=9$. This excludes the cases $p=3$ and $n=6$ or $=12$.

6.2. The special cases. Just as before we denote by $V \subset N S(Y)$ the sublattice spanned by the rational curves from sect. 4.1. Now it has rank 20 . In the same way, as in sect. 6.1 we check, that for $n=6$ the classes $L / 3, L^{\prime} / 3$ and for $n=8$ the classes $L / 2, L^{\prime} / 2$ in $N S(Y)$ exist. Intersecting with the twentieth rational curve we find, that the curves can be labelled as in the diagrams of sect. 4.2.

THEOREM 6.2. In all cases $N S(Y)$ is spanned by the classes from sect. 6.1 and the twentieth rational curve. The discriminants of the lattices are

\begin{tabular}{c|cccc|cccc} 
case & 6,1 & 6,2 & 6,3 & 6,4 & 8,1 & 8,2 & 8,3 & 8,4 \\
\hline$d$ & -15 & -60 & -60 & -15 & -28 & -84 & -168 & -112 \\
& & & & & & & & \\
& & case & 12,1 & 12,2 & 12,3 & 12,4 \\
\hline & $d$ & -660 & -1320 & -792 & -132
\end{tabular}


Proof. Denote by $C$ the twentieth rational curve and by $W$ the lattice spanned by $V$ and $C$. The discriminants in the table above are those of the lattice $W$. We have to show $W=N S(Y)$. If this would be not the case, there would be a lattice $W^{\prime}$ with $W \subset W^{\prime} \subset N S(Y)$ such that $p:=\left[W^{\prime}: W\right]$ is a prime with $p^{2}$ dividing $d(W)$. In the following table we collect the possibilities and give in each case generators for the $p$-subgroup $\left(W^{\vee} / W\right)^{p}$ of $W^{\vee} / W$. (The cases 6,2 and 6,3 are essentially the same.)

\begin{tabular}{rr|l} 
case & $p$ & generators \\
\hline 6,2 & 2 & $M_{1} / 2,\left(N_{1}+C+N_{4}\right) / 2$ \\
\hline 8,1 & 2 & $\left(M_{1}+M_{2}+M_{4}\right) / 2,\left(M_{3}+M_{4}+R_{1}+R_{3}\right) / 2$ \\
8,2 & 2 & $\left(M_{1}+M_{2}+M_{3}\right) / 2,\left(M_{1}+M_{2}+M_{4}\right) / 2$ \\
8,3 & 2 & $\left(M_{1}+M_{2}+M_{4}\right) / 2,\left(M_{1}+M_{2}\right) / 2+\left(R_{1}+2 R_{2}+3 R_{4}\right) / 4$ \\
8,4 & 2 & $\left(M_{1}+M_{2}\right) / 2+\left(2 N_{1}+2 C+M_{3}+3 M_{4}\right) / 4$, \\
& & $\left(M_{1}+M_{2}\right) / 2+\left(R_{1}+2 R_{2}+3 R_{3}\right) / 4$ \\
\hline 12,1 & 2 & $M_{2} / 2, M_{3} / 2$ \\
12,2 & 2 & $M_{3} / 2,\left(2 N_{1}+2 C+M_{1}+3 M_{2}\right) / 4$ \\
12,3 & 2 & $M_{3} / 2,\left(2 S_{1}+2 S_{3}+2 C+M_{1}+3 M_{2}\right) / 4$ \\
& 3 & $\left(N_{1}-N_{2}\right) / 3,\left(N_{3}-N_{4}\right) / 3$ \\
12,4 & 2 & $M_{2} / 2, M_{3} / 2$
\end{tabular}

In each single case there are not enough rational curves to meet the conditions $[\mathrm{N}]$ for a divisor divisible by 2 or [T] for a divisor divisible by 3 .

7. Comments. 1) Denote by $M_{k}$ the moduli-space of abelian surfaces with level$(1, \mathrm{k})$ structure In $[\mathrm{Mu}]$ the quotients $\mathbb{P}_{3} / G_{6}$, resp. $\mathbb{P}_{3} / G_{8}$ are identified with the Satake-compactification of $M_{3}$, resp $M_{4}$, and $\mathbb{P}_{3} / G_{12}$ is shown to be birationally equivalent with the Satake-compactification of $M_{5}$. However the proof there is not very explicit. It is desirable to have an explicit identification of the quotient $\mathbb{P}_{3} / G_{n}$ with the corresponding moduli space. The pencil $Y_{\lambda}^{\prime}$ on $\mathbb{P}_{3} / G_{n}$ might be useful.

2) We did not consider the quotient threefold $\mathbb{P}_{3} / G_{n}$. We just identified the minimal non-singular model $Y_{\lambda}$ for each quotient $Y_{\lambda}^{\prime}$. Of course it would be desirable to have a global resolution of $\mathbb{P}_{3} / G_{n}$ and to view our K3-surfaces as a pencil on this smooth threefold. One would need a particular crepant resolution of the singularities of $\Upsilon$. Such resolutions are given e.g. in [I, IR, Ro]. We would need a resolution, where the behaviour of the K3-surfaces can be controlled, to identify the partial resolutions of the four special surfaces.

3) Our quotient surfaces admit a natural involution induced by the symmetry $C$ from [S, p. 433] normalizing $G_{n}$, but not belonging to $S L(4, \mathbb{C})$. It would be interesting to identify the quotients.

4) By $[\mathrm{Mo}]$ each K3-surface with Picard number 19 admits a Nikulin-involution, an involution with eight isolated fix-points. We do not know how to identify it in our cases. It cannot be the involution from 3), because this has a curve of fix-points. It is also not clear to us, whether this Nikulin-involution exists globally, i.e. on the total space $\Upsilon$ of our fibration. This Nikulin-involution is related to the existence of a sub-lattice $E_{8} \perp E_{8} \subset N S(Y)$. We did not manage to identify such a sub-lattice.

5) It seems remarkable that the Picard group of the general surface in a pencil of K3-surfaces can be identified so explicitly, as it is done in sect. 6. It is also remarkable that the quotient K3-surfaces have Picard number $\geq 19$. Such pencils have been studied in [Mo] and [STZ]. We expect our surfaces to have some arithmetical meaning. In particular the prime factor $n-1=5,7,11$ in the discriminant of the Picard lattices draws attention. In fact, the same prime factor appears in each polynomial 
$s_{n}, n=6,8,12$ from [S]. It can be found too in the cross-ratio $C R\left(\lambda_{1}, \ldots, \lambda_{4}\right)$ of the four special parameters in each pencil $X_{\lambda}$ and together with strange prime factors in the absolute invariant $j$ :

\begin{tabular}{c|ccc}
$n$ & 6 & 8 & 12 \\
\hline$C R$ & $\frac{5^{2}}{3^{2}}$ & $\frac{7^{2}}{2^{4} \cdot 3}$ & $\frac{11^{2}}{2^{5} \cdot 3}$ \\
$j$ & $\frac{13^{3} \cdot 37^{3}}{2^{8} \cdot 3^{4} \cdot 5^{4}}$ & $\frac{13^{3} \cdot 181^{3}}{2^{8} \cdot 3^{2} \cdot 7^{4}}$ & $\frac{12241^{3}}{2^{10} \cdot 3^{2} \cdot 5^{4} \cdot 11^{4}}$
\end{tabular}

\section{REFERENCES}

[B] Barth, W., K3-surfaces with nine cusps, Geom. Dedic., 72 (1998), pp. 171-178

[BPV] Barth, W., Peters, C., Van de Ven A., Compact Complex Surfaces, Ergebn. Math (3), 4, Springer 1984.

[C] COXETER, H.S.M., The product of the generators of a finite group generated by reflections, Duke Math. J., 18 (1951), pp. 765-782.

[FG] Fischer, W., GrauerT, H., Lokal triviale Familien kompakter komplexer Mannigfaltigkeiten, Nachr. Akad. Wiss. Göttingen II Math. Phys. Kl. (1965), pp. 89-94.

[I] Iто, Y., Crepant resolutions of trihedral singularities, Proc. J. Acad. Sci., 70 (1994), Ser A, pp. 131-136.

[IR] Ito, Y., Reid, M., The McKay correspondence for finite subgroups of $S L(3, \mathbb{C})$, In: Higher Dimensional Complex Varieties, Proc. Conf. Trento 1994, pp. 221-240.

[Mu MukaI, S., Moduli of abelian surfaces and regular polyhedral groups, In: Proc. of Moduli of Algebraic Varieties Symposium, Sapporo (1999).

[Mo] Morrison, D., On K3-surfaces with large Picard number, Inv. math., 75 (1984), pp. 105121.

[N] Nikulin, V., On Kummer surfaces, Izv. Akad. Nauk. SSSR, Ser. Math., 39 (1975), pp. $1145-1170$.

[Ra] RACAH, G., Sulla caratterizzatione delle rappresentazioni irriducibili dei gruppi semisemplici di Lie, Rend. Accad. Naz. Linc, Sci. Fis. Mat. Nat (8), 8 (1950), pp. 108-112.

[Ro] RoAN, S.S., Minimal resolutions of gorenstein orbifolds in dimension three, Topology, 35 (1996), pp. 489-508.

[S] Sarti, A., Pencils of symmetric surfaces in $\mathbb{P}_{3}$, J. of Alg., 246 (2001), pp. 429-552.

[SI] Shioda, T., Inose H., On singular K3-surfaces, In: Complex Analysis and Algebraic Geometry, Iwanami-Shoten, Tokyo (1977), pp. 117-136.

[STZ] Sun, X., TAN, S.-L., Zuo, K., Families of K3-surfaces over curves satisfying the equality of Arakelov-Yau's type and modularity, Preprint (2002).

[T] TAN, S.-L., Cusps on some algebraic surfaces and plane curves, Preprint (1999). 